\title{
Hip Fractures with Rotational Instability: Concept and Surgical Technique
}

\section{Aguado-Maestro I*, Escudero-Marcos R, Nistal-Rodríguez J, Alonso-García N, Pérez-Bermejo D, Bañuelos-Díaz A, Cebrián-Rodríguez E, Ramos-Galea $R$, and García-Alonso $M$}

Hospital Universitario del Río Hortega, Valladolid, Spain

\begin{abstract}
Introduction: New concepts regarding instability in extra capsular fractures have led to a better understanding of their biomechanical behavior. Location and direction of the fracture line determine rotational instability in typically defined "stable" fractures. We propose a modification of the classical osteosynthesis with endomedular nail.

Material and methods: Fourteen patients met the inclusion criteria (31A1 fractures with a fracture line angled more than 70 degrees) between October 2012 and February 2013. They were all treated with PFNA nail, standard technique modified by adding a $7.3 \mathrm{~mm}$ cannulated screw with anti-rotation effect as described by the authors. X-Ray controls were performed after 24 hours, and after 1, 3 and 6 months, evaluating reduction, helical blade position (Cleveland-Bosworth) and tip-apex distance (Baumgaertner). Consolidation, loss of reduction and function were also reviewed.
\end{abstract}

Results: Reduction quality was good or fair in $91 \%$, founding the helical blade in center-center position in every case, with an average tip-apex distance of $12.24 \mathrm{~mm}$.

Conclusions: The theory of helical blade rotation through the femoral is becoming more and more relevant regarding cut out. Defining those fracture lines affected by rotational instability will lead to more easily determining the criteria for using a cannulated screw for additional stabilization. The technique is safe, simple and easily replicated. Satisfactory results were observed in all the cases, despite the small sample size.

Keywords: Hip fracture; Rotational instability; Surgical technique

\section{Introduction}

The high occurrence of hip fractures among senior citizens cannot be overlooked, as they are the most frequent cause of admission to trauma units in cases involving elderly patients. Half of them are considered extra capsular, leading to high mortality rates.

The endomedular nail is one of the most frequently used devices for the treatment of these fractures in our hospitals at the moment. This device allows a shorter moment arm due to the force being applied over a shorter distance. This reduces bending loads seen by the implant. Helical blade in Proximal Femoral Nail Antirotation provides in addition greater angular and rotational stability by compacting trabecular bone while being inserted. Other systems have been designed to prevent rotation of the cervical fragment, such us double-screw nails. However, helical blade itself showed a decrease in postoperative implant-associated complications, especially in osteoporotic bone and unstable fracture types [1].

It has been demonstrated that intramedullary nails are better than sliding hip screws in unstable fractures [2-4]. Such unstable patterns consist of reverse obliquity fractures, transtrochanteric fractures, fractures with a large posteromedial fragment implying loss of the medial buttress, and fractures with subrochanteric extension $[4,5]$.

Osteoporosis was considered by Harrington to be a predictor for instability in 1975 [6]. Recent studies have dispelled this theory. The relationship between the Singh method for radiographic evaluation of osteoporosis and mechanical complications remains controversial $[7,8]$. Furthermore, there is a wide variability intra and interobserver in Singh's classification [9].

The most common classification for these fractures is the provided by AO/OTA, which divides the patterns into 3 different groups; 1 (pertrocantheric simple), 2 (pertrocantheric multifragmentary), and 3 (intertrocantheric) [10]. This is, for the most part, an anatomical classification. However, in previous works [11], we noticed a higher incidence of cut out in simple pattern (AO/OTA 31A1) basicervical fractures treated with endomedullary nail (Proximal Femoral Nail
Antirotation), which involved the 4 cases we had. In these four cases, we appreciated a simple fracture line, angled in more than 70 degrees with an imaginary horizontal line.

A basicervical fracture is one that extends through the base of the femoral neck, next to the intertrochanteric region [12]. It is postulated that rotation of the femoral head is a potential initiator for cutting out $[13,14]$. Previous works by Massoud considered the possibility of treating these fractures with a DHS using a derotation screw.

The aim of this article is to present the surgical technique and results of basicervical fractures treated in our hospital with a Proximal Femoral Nail Antirotation with a derotation screw to enhance rotational stability.

\section{Material and Methods}

Fourteen consecutive patients met the inclusion criteria between October 2012 and February 2013. These criteria consisted of basicervical fractures (AO/OTA 31A1) with a fracture line angled at more than 70 degrees against an imaginary horizontal line, low-energy trauma (self height fall) and osteoporosis. The exclusion criteria excluded patients aged 60 years old or less and high-energy traumas.

There were 14 women, with an average age of 80.3 years old (6487). The evaluation of the results was done by an independent surgeon. All of them were treated with a PFNA nail with a modified technique

*Corresponding author: Aguado-Maestro I, Hospital Universitario del Río Hortega, Valladolid, Spain, E-mail: nacho.ams@gmail.com

Received August 03, 2013; Accepted August 28, 2013; Published September 07, 2013

Citation: Aguado-Maestro I, Escudero-Marcos R, Nistal-Rodríguez J, AlonsoGarcía N, Pérez-Bermejo D, et al. (2013) Hip Fractures with Rotational Instability: Concept and Surgical Technique. Surgery Curr Res 3: 146. doi:10.4172/2161 1076.1000146

Copyright: (c) 2013 Aguado-Maestro I, et al. This is an open-access article distributed under the terms of the Creative Commons Attribution License, which permits unrestricted use, distribution, and reproduction in any medium, provided the original author and source are credited. 
by adding a $7.3 \mathrm{~mm}$ cannulated derotation screw as described below. $\mathrm{X}$-Ray controls were performed 24 hours after surgery and during clinical follow-ups (1, 3 and 6 months post-op).

All patients were operated on as soon as possible. One single senior orthopedic surgeon performed the surgery, with the assistance of a resident, usually within the first 48 hours. Subarachnoid anesthesia was preferred, although general anesthesia was used in patients treated with clopidogrel. Infection prophylaxis was made with cefazolin (unless allergic to betalactamics) and thromboprophylaxis was made with bemiparin. Sitting was permitted (unless in cases of medical contraindication) in 24 hours after blood and radiological tests. Ambulation was permitted in every case between 72 and 96 hours. These permissions are not dependant on the type of fracture or reduction quality $[15,16]$.

Follow up revisions were scheduled at 1, 3, 6 and 12 months after surgery in the clinics at our hospital. A comparison between the previous ambulation capacity and the best ambulation achieved during follow up is made and is classified in one of the following groups [8]: group 1 (walks without help or with a single and simple aid), group 2 (walks aided by 2 English canes or a walking frame), group 3 (unable to walk, needs the assistance of a wheel-chair). During follow up, all patients started treatment with Calcium and Vitamin D, adding ibandronic acid once consolidation was achieved.

Reduction quality was defined taking into account the neck-shaft angle in the anterioposterior view, and the reduction gap, which should be less than $5 \mathrm{~mm}$ both in AP and axial views. Helical blade and derotation screw's position was evaluated according to ClevelandBosworth's division of the femoral head [17] and tip-apex distance as described by Baumgaertner [18]. Consolidation, loss of reduction and function were also reviewed.

\section{Surgical Technique}

We've made a modification of the standard PFNA (DePuySynthes) technique. The patient is positioned supine on an extension table and the fracture reduced under C-arm control. Antirotation wires may be used as usual but should be removed before intraoperative compression in order to make it possible, as the $\mathrm{K}$ wire used for antirotation purpose and the $\mathrm{K}$ wire used to guide the helical blade are not necessarily parallel.

Once compression is performed, we use the aiming jig for antirotation wires and the drill sleeve. Usually in superior-anterior position to the aiming arm. It is important not to use the provided $\mathrm{K}$-wires with the PFNA instrumental, as they are not thin enough for the $7.3 \mathrm{~mm}$ cannulated screws. We prefer to use the $\mathrm{K}$-wires provided with the screws. Once inserted the K-wire, we disengage the aiming jig to measure the screw length. For this purpose, we usually use a samelength wire and measure the difference. After drilling, the cannulated derotation screw is inserted (Figures 1 and 2).

\section{Results}

All fractures were classified ad AO31A1 according to our inclusion criteria. The nails used for the osteosynthesis are summarized in the chart below (Table 1) the most common being $200 \mathrm{~mm}$ length, 130 neck-shaft degrees, $10 \mathrm{~mm}$ in diameter. In every case a distal dynamic locking was done. Average surgery time was $50 \mathrm{~min}$ ( 8 minutes more than the average time achieved without using a derotation screw) [11] $(\mathrm{p}=0,05)$

Quality of reduction was considered as 'good' (both angulation and reduction gap objectives were achieved) in 50\% and 'fair' (only one of the criteria was met) in $50 \%$.

Center-center helical blade position was achieved in all cases. Cannulated screw positions are represented in (Figure 3). Average tip-apex distance, which should be under $25 \mathrm{~mm}$ to prevent cut-out [2,9,18-21] was $12.24 \mathrm{~mm}$ [9.51-14.07 mm]. Derotation screw's tipapex distance was $21.21 \mathrm{~mm}$ [13.18-32.22 mm].

The average hospital stay was 9 days (5-14 days) (slightly shorter than the average hospital stay in our previous study [11], which was 9 ,
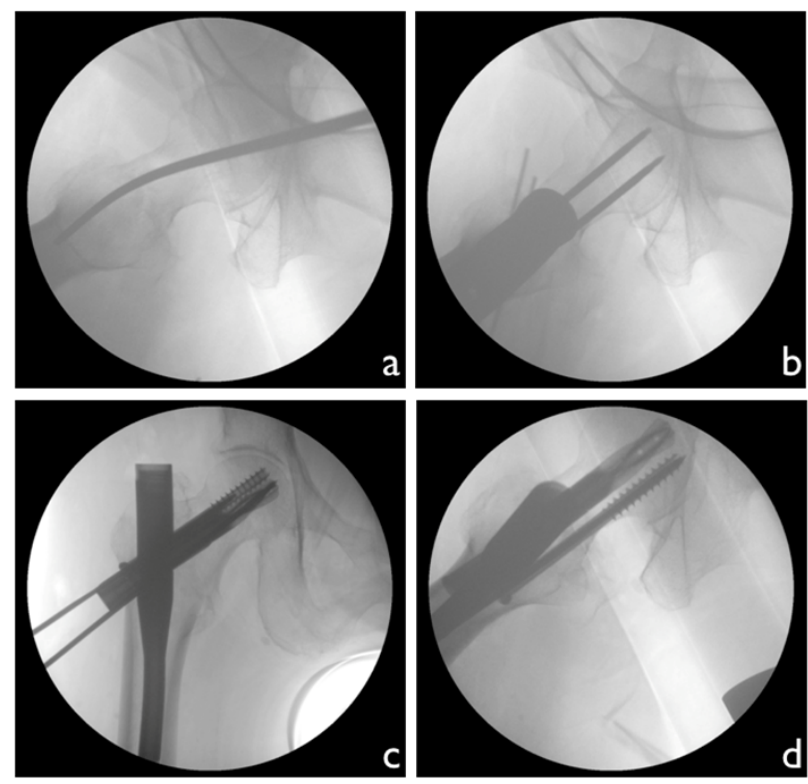

Figure 1: Surgical technique (a) Entry point at the tip of the greater trochanter in the center of the channel, assessed by an axial X-Ray (b) K-wires position, axial view (c) Helical blade and cannulated derotation screw, AP view (d) Axial view.
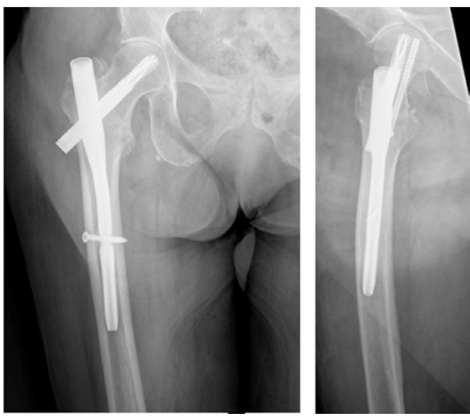

Figure 2: X Ray controls after 1 month of follow-up

\begin{tabular}{|c|c|c|c|c|c|}
\hline PFNA length & $170 \mathrm{~mm}$ & $200 \mathrm{~mm}$ & $240 \mathrm{~mm}$ & & \\
\hline & 1 & 13 & 0 & & \\
\hline PFNA angle & $130^{\circ}$ & & & & \\
\hline & 14 & & & & \\
\hline Helical blade & $80 \mathrm{~mm}$ & $85 \mathrm{~mm}$ & $90 \mathrm{~mm}$ & $95 \mathrm{~mm}$ & $110 \mathrm{~mm}$ \\
\hline & 2 & 5 & 0 & 5 & 2 \\
\hline Cannulated screw & $70 \mathrm{~mm}$ & $85 \mathrm{~mm}$ & $95 \mathrm{~mm}$ & $110 \mathrm{~mm}$ & \\
\hline & 2 & 6 & 4 & 2 & \\
\hline
\end{tabular}

Table 1: Distribution of the components employed for the osteosynthesis in the study. 


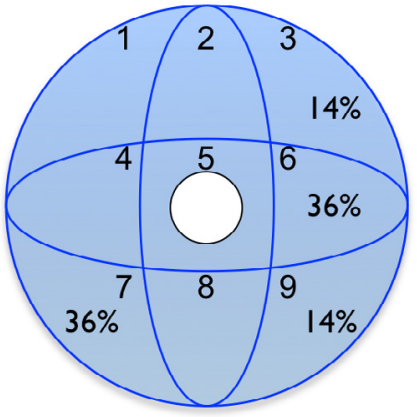

Figure 3: Distribution in the areas of Cleveland and Bosworth of the helical blade (represented in white, 100\%) and the cannulated derotation screw.

17 days $(\mathrm{p}>0,05)$. The average delay between hospital admission and surgery was 3.33 days (1-7 days).

Follow up time ranged between 6 and 10 months. No loss to followup was observed.

Functional ability was measured before and after surgery. Before surgery, 7 patients (50\%) belonged to group 1 (walked without help or with a simple aid, as a cane, and 7 patients (50\%) belonged to group 2 (walked aided by 2 English canes or a walking frame). After surgery, 14 patients (100\%) belonged to group number 2 (all of them using walking frame, with the exception of 2 patients that used two English canes). No patients needed wheelchair after surgery.

No complications were observed in our series. There was one only case in which the fracture collapsed $5 \mathrm{~mm}$, observing bone healing one month later. Consolidation was achieved in 14 patients (100\%).

\section{Discussion}

Mechanical complications are the most frequent type of complications. They lead to implant failure and the need for further surgical intervention. Factors which condition these complications may be surgeon-dependent (Tip-Apex Distance, helical blade position, quality of reduction, implant election) or surgeon non-dependent (mineral density, type of fracture).

The development of new systems for osteosynthesis and a more accurate surgical technique has helped to reduce the mechanical complications rate.

In our previous study, basicervical fractures grouped most of the mechanical complications related to cut-out, which led us to consider other causes rather than helical blade position in the femoral head. It's rotation, favored by the lack of muscular insertions which stabilize the fracture, may be produced both during the insertion of the helical blade and during ambulation. There are new theories which assert that torque forces acting against the helical blade during ambulation may lead to trabecular breakage next to the implant, permitting head rotation [13]. Surgical techniques such as augmentation or derotation screws were designed to prevent these complications.

Radiographic evaluation of osteoporosis according to Singh's method was not done due to the controversy regarding the relationship between mechanical complications and Singh's trabecular pattern $[7,8]$.

At the moment, PFNA nail is the fixation method for the treatment of stable and unstable trochanteric fractures, including those affecting the basicervical line. This system has better mechanical properties and a lesser inflexion moment than the Dynamic Hip Screw. Helical blade is thought to add angular and rotational stability to the complex, as it compacts cancellous bone while being inserted. It's sliding capacity favors the compression of the fracture $[22,23]$.

Different nails have been designed to prevent rotation. Doublescrew designs have shown the appearance of a new kind of complication, the so-called "Z-Effect" [23-26]. The Z-effect is a potential complication of two lag screw intramedullary nail designs, in which the inferior lag screw migrates laterally and the superior lag screw migrates medially during loading, which may lead to failure of the osteosynthesis. The helical blade has demonstrated to be superior to double-screw nails osteosynthesis [1]. Unfortunately, there are no double-screw systems with a helical blade design. However, different complications have appeared regarding basicervical fractures. These specific kinds of fractures have a special rotational instability which may lead to implant failure, despite its design. The use of a derotation cannulated screw adds rotational stability, without the appearance of the Z-effect, as the screw is not attached to the nail.

The results of the study prove that the addition of a derotation cannulated screw is a reproducible technique, without major complications. It can be done with an increase of only 9 minutes of surgical time, thanks to the PFNA instrumentation. However, we cannot conclude at this time that there is a significant difference in the prevention of cutting out, due to the small sample size.

The shorter hospital stay, may be related to the lack of early complications seen in our previous study, such us deep vein thrombosis, pneumonia or pulmonary embolisms.

The expected incidence of complications, according to our previous study [11] was high (7,5\% regarding al 31A fractures, rising to more than $20 \%$ taking into account only basicervical fractures). The results of the study suggest that using a derotation cannulated screw may prevent mechanical complications. It is not possible to establish a statistical comparison between these outcomes, due to the differences in other variables (such as helical blade position, which has been centre-centre in all cases of this study).

The strength of the study is related to the performance of every intervention by the same senior surgeon, preventing the different outcome achieved by different surgeons while implementing the new technique. Secondly, no loss to follow-up prevents the apparition of bias. Finally, follow up during more than 6 months in every case let us assured us that there will not be any late complication undocumented, as most of them usually occur during the first 6 months after surgery.

There are limitations such as the kind of study (retrospective) and the small sample size. Variables not reflected in the clinical histories cannot be studied. A bigger sample size would have allowed us to ascertain any other potential complications, as their rate of occurrence is low.

\section{References}

1. Penzkofer J, Mendel T, Bauer C, Brehme K (2009) [Treatment results of pertrochanteric and subtrochanteric femoral fractures: a retrospective comparison of PFN and PFNA]. Unfallchirurg 112: 699-705.

2. Haidukewych GJ, Israel TA, Berry DJ (2001) Reverse obliquity fractures of the intertrochanteric region of the femur. J Bone Joint Surg Am 83-83A: 643-50.

3. Bridle SH, Patel AD, Bircher M, Calvert PT (1991) Fixation of intertrochanteric fractures of the femur. A randomised prospective comparison of the gamma nail and the dynamic hip screw. J Bone Joint Surg $\mathrm{Br} 73$ : 330-334.

4. Sadowski C, Lübbeke A, Saudan M, Riand N, Stern R, et al. (2002) Treatmen 
Citation: Aguado-Maestro I, Escudero-Marcos R, Nistal-Rodríguez J, Alonso-García N, Pérez-Bermejo D, et al. (2013) Hip Fractures with Rotational Instability: Concept and Surgical Technique. Surgery Curr Res 3: 146. doi:10.4172/2161-1076.1000146

of reverse oblique and transverse intertrochanteric fractures with use of an intramedullary nail or a 95 degrees screw-plate: a prospective, randomized study. J Bone Joint Surg Am 84-84A: 372-81.

5. Haidukewych GJ (2009) Intertrochanteric fractures: ten tips to improve results. J Bone Joint Surg Am 91: 712-719.

6. Harrington KD (1975) The use of methylmethacrylate as an adjunct in the internal fixation of unstable comminuted intertrochanteric fractures in osteoporotic patients. J Bone Joint Surg Am 57: 744-750.

7. Singh M, Nagrath AR, Maini PS (1970) Changes in trabecular pattern of the upper end of the femur as an index of osteoporosis. J Bone Joint Surg Am 52 457-467.

8. Herman A, Landau Y, Gutman G, Ougortsin V, Chechick A, et al. (2012) Radiological evaluation of intertrochanteric fracture fixation by the proximal femoral nail. Injury 43: 856-863.

9. Koot VC, Kesselaer SM, Clevers GJ, de Hooge P, Weits T, et al. (1996) Evaluation of the Singh index for measuring osteoporosis. J Bone Joint Surg $\mathrm{Br}$ 78: 831-834.

10. Marsh JL, Slongo TF, Agel J, Broderick JS, Creevey W, et al. (2007) Fracture and dislocation classification compendium - 2007: Orthopaedic Trauma Association classification, database and outcomes committee. J Orthop Trauma 21: S1-133.

11. Aguado-Maestro I, Escudero-Marcos R, García-García J M, Alonso-García N, Pérez-Bermejo, et al. (2013) Results and complications of pertrochanteric hip fractures using an intramedullary nail with a helical blade (proximal femoral nail antirotation) in 200 patients. Revista Española de Cirugía Ortopédica y Traumatología 57: 201-207.

12. Blair B, Koval KJ, Kummer F, Zuckerman JD (1994) Basicervical fractures of the proximal femur. A biomechanical study of 3 internal fixation techniques. Clin Orthop Relat Res: 256-263

13. Lenich A, Bachmeier S, Prantl L, Nerlich M, Hammer J, et al. (2011) Is the rotation of the femoral head a potential initiation for cutting out? A theoretical and experimental approach. BMC Musculoskelet Disord 12: 79.

14. Massoud El (2010) Fixation of basicervical and related fractures. Int Orthop 34: $577-582$
15. Koval KJ, Sala DA, Kummer FJ, Zuckerman JD (1998) Postoperative weightbearing after a fracture of the femoral neck or an intertrochanteric fracture. $J$ Bone Joint Surg Am 80: 352-356.

16. Koval KJ, Friend KD, Aharonoff GB, Zukerman JD (1996) Weight bearing after hip fracture: a prospective series of 596 geriatric hip fracture patients. J Orthop Trauma 10: 526-530.

17. Cleveland M, Bosworth DM, Thompson FR, Wilson HJ Jr, Ishizuka T (1959) A ten-year analysis of intertrochanteric fractures of the femur. J Bone Joint Surg Am 41A: 1399-408.

18. Baumgaertner MR, Curtin SL, Lindskog DM, Keggi JM (1995) The value of the tip-apex distance in predicting failure of fixation of peritrochanteric fractures of the hip. J Bone Joint Surg Am 77: 1058-1064.

19. PFNA [surgical technique. Solothurn, Switzerland: Synthes $\mathrm{GmbH}$.

20. Frei HC, Hotz T, Cadosch D, Rudin M, Käch K (2012) Central head perforation, or "cut through," caused by the helical blade of the proximal femoral nail antirotation. J Orthop Trauma 26: e102-107.

21. Baumgaertner MR, Solberg BD (1997) Awareness of tip-apex distance reduces failure of fixation of trochanteric fractures of the hip. J Bone Joint Surg $\mathrm{Br} 79$ 969-971.

22. Brunner A, Jöckel JA, Babst R (2008) The PFNA proximal femur nail in treatment of unstable proximal femur fractures--3 cases of postoperative perforation of the helical blade into the hip joint. J Orthop Trauma 22: 731-736.

23. Simmermacher RK, Ljungqvist J, Bail H, Hockertz T, Vochteloo AJ, et al. (2008) The new proximal femoral nail antirotation (PFNA) in daily practice: results of a multicentre clinical study. Injury 39: 932-939.

24. Strauss EJ, Kummer FJ, Koval KJ, Egol KA (2007) The "Z-effect" phenomenon defined: a laboratory study. J Orthop Res 25: 1568-1573.

25. Pires RE, Santana EO Jr, Santos LE, Giordano V, Balbachevsky D, et al. (2011) Failure of fixation of trochanteric femur fractures: Clinical recommendations for avoiding Z-effect and reverse Z-effect type complications. Patient Saf Surg 5 17.

26. Akan K, Cift H, Ozkan K, Eceviz E Tasyikan L, et al. (2011) Effect of osteoporosis on clinical outcomes in intertrochanteric hip fractures treated with a proximal femoral nail. J Int Med Res 39: 857-865. 